\title{
Measurement of Fractional Exhaled Nitric Oxide as a Marker of Disease Activity in Inflammatory Bowel Disease
}

\author{
Erkanda Ikonomi ${ }^{1}$, Robin D. Rothstein ${ }^{2}$, Adam C. Ehrlich ${ }^{2 *}$, Frank K. Friedenberg ${ }^{2}$ \\ ${ }^{1}$ Department of Medicine,Temple University School of Medicine, Philadelphia, PA. \\ ${ }^{2}$ Temple Inflammatory Bowel Disease Program, Temple University School of Medicine, Philadelphia, PA
}

Received: October 22, 2015; Accepted: January 15, 2016; Published: January 27, 2016

*Corresponding author: Adam C. Ehrlich, MD, MPH, Assistant Professor of Medicine, Gastroenterology Section, Temple University School of Medicine, 3401 North Broad Street, Philadelphia, PA 19140, Phone: 215-707-9900; Fax: 215-707-2684; Email: adam.ehrlich@tuhs.temple.edu

\begin{abstract}
Background and Aims: Definitive diagnosis of IBD requires endoscopic and pathologic confirmation. These tools are also used to classify disease activity. Our aim was to determine if the fractional exhaled nitric oxide (FeNO) could be utilized to screen for IBD and assess for disease activity.

Methods: We matched weighted IBD cases and controls from the 2009-2010 NHANES dataset. All subjects underwent measurement of FeNO using standardized techniques. We assessed for potential confounders for FeNO measurement including age, height, and asthma. For IBD subjects, we used the presence of diarrhea, fatigue, and weight loss as a proxy for IBD activity. Laboratory parameters examined to estimate disease activity included anemia $(\leq 10 \mathrm{~g} / \mathrm{dl})$, iron deficiency (ferritin $\leq 20 \mathrm{ng} / \mathrm{ml}$ ), hypoalbuminemia $(\leq 3.2 \mathrm{~g} / \mathrm{dl}$ ), and CRP ( $\geq 1.1 \mathrm{mg} / \mathrm{dl})$.

Results: The weighted sample represented 199,414,901 subjects. The weighted prevalence of IBD was $2,084,895$ (1.0\%). IBD subjects had nearly the same FeNO level as those without IBD (17.0 \pm 16.2 vs. $16.7 \pm 14.5 \mathrm{ppb}$ ). The odds of a FeNO $>25$ ppb was half (OR $=0.501 ; 95 \%$ CI 0.497-0.504) for subjects with IBD compared to those without IBD after controlling for confounders. The AUROC curve for FeNO was 0.47 (0.35-0.59). FeNO levels were not higher in patients with laboratory values suggestive of active disease. FeNO levels were higher in IBD patients with diarrhea, rectal urgency, and fatigue but were lower in those with unintentional weight loss.
\end{abstract}

Conclusion: Measurement of FeNO does not appear to be useful to screen for IBD or assess disease activity.

Keywords: Biomarker; Crohn's disease; Inflammation; Inflammatory bowel disease; Nitric oxide; Ulcerative colitis

\section{Abbreviations}

IBD: Inflammatory bowel disiease; CD: Crohn's Disease; CDAI: Crohn's Disease Activity Index; CRP: C-reactive protein; ESR: erythrocyte sedimentation rate; FENO: fractional exhaled nitric oxide; NHANES: National Health and Nutrition Examination Survey; ROC: receiver operator characteristic

\section{Introduction}

Inflammatory bowel disease (IBD) is a chronic inflammatory disorder. In ulcerative colitis (UC), the inflammation is confined to the colon mucosal layer, while in Crohn's disease (CD), the inflammation is transmural and may involve the entire gastrointestinal tract [1]. Medical and surgical management of IBD is primarily driven by an accurate assessment of disease activity. The gold standard tools used to assess disease activity are cross-sectional imaging and mucosal inspection via endoscopy. Unfortunately, these tests are costly, cumbersome to use serially, and have the potential for adverse events. For many years, clinicians have relied on non-invasive tests to estimate disease activity. For example, laboratory tests may reveal iron deficiency anemia. Elevations of the erythrocyte sedimentation rate (ESR) and/or C-reactive protein (CRP) often correlate with disease activity although either may be normal in the setting of active disease. Studies suggest that CRP is the most sensitive marker in detecting active IBD but values range from $50 \%$ to $60 \%$ for UC and $70 \%$ to $100 \%$ for CD, depending on the cutoff value used [2]. Recently, evaluation of stool concentrations of lactoferrin and calprotectin has become routine with the commercial availability of kits for their measurement. D'Haens, et al., using a cutoff value for calprotectin of $250 \mu \mathrm{g} / \mathrm{g}$ of stool, found the presence of active colonic CD with a sensitivity of $60.4 \%$ and a specificity of $79.5 \%$ and active UC with a sensitivity of $71.0 \%$ and specificity of $100.0 \%$ [3].

A new potential non-invasive marker of IBD disease activity is nitric oxide (NO). NO is produced from L-arginine by three nitric oxide synthases (NOS), including endothelial, neuronal and inducible subtypes. The former two are constantly active in endothelial cells and neurons, while the latter can be activated in response to microbes, cytokines, and other stimuli in many cell types including epithelial cells [4]. Colonic NO can be measured using a chemiluminescence technique [5]. One study demonstrated that colonic NO levels in six adults with UC were 100 -fold higher compared to twelve controls [6]. Similar results have been reported in children where it was found that those with active UC and CD had greatly increased rectal NO concentrations compared with controls $(8,840 \pm 5,120$ and $15,170 \pm 4,757$ vs. 77 \pm 17 ppb; $\mathrm{P}<0.001$ ) [5]. Children with inactive UC and CD had levels in between (356 \pm 110 and $188 \pm 55$ ppb respectively). For 
reference, the ambient concentration of NO in room air is 5-10 $\mathrm{ppb}[6]$.

One non-invasive approach to measuring NO production from mucosal surfaces is to calculate the fractional concentration of exhaled nitric oxide (FeNO). The most widely used technique is a chemiluminescence reaction [7]. FeNO is already in use to quantify airway inflammation, and increased levels have been reported in asthma and acute exacerbations of chronic obstructive pulmonary disease [8]. In asthma, elevation is typically found in the setting of eosinophil-driven inflammation [9]. Eosinophilic airway inflammation results from the activation of mast cells and antigen-specific Th2 cells resulting in the production of cytokines and up-regulation of epithelial inducible NO [10].

A recent study reported a significant increase in exhaled FeNO levels (median, [interquartile range]) in steroid-free CD patients with clinically active $(n=16)$ disease (Crohn's Disease Activity Index (CDAI) $>150 ; 22$ [8] ppb) compared with $\mathrm{CD}$ patients $(\mathrm{n}=34)$ in clinical remission $(\mathrm{CDAI}<150 ; 11[6] \mathrm{ppb} ; \mathrm{P}<0.001)$ and healthy $(\mathrm{n}=25)$ controls $(17$ [9] ppb; $\mathrm{P}<0.05)$ [11]. The authors found a correlation $(\mathrm{r}=0.68)$ between FeNO and the CDAI but no correlation with fecal calprotectin.

Koek, et al. performed FeNO measurements on patients with CD ( $\mathrm{n}=31)$, UC $(\mathrm{n}=24)$ and healthy non-smoking controls $(n=27)$. CD and UC patients had mild activity based on CDAI and Ulcerative Colitis Activity Index scores. For CD patients their mean FeNO level was $13.5 \pm 4.6 \mathrm{ppb}$ and for UC the level was 15.8 $\pm 6.2 \mathrm{ppb}$. In healthy controls the FeNO was significantly lower at $10.2 \pm 2.5 \mathrm{ppb}(\mathrm{P}<0.05$ compared to $\mathrm{CD}$ and $\mathrm{P}<0.01$ compared to UC) [12]

Two theories have been proposed to explain the correlation between FeNO levels and IBD activity although the underlying mechanism is poorly understood. One proposed mechanism is that both colonic and respiratory epithelium originate from primitive foregut and include goblet cells, submucosal glands and lymphoid tissue that play a role in host mucosal defense. Secondly, an injury to the intestinal barrier due to circulating immune complexes in IBD may result in an entry of antigens that can induce both local and systemic inflammation [13].

Our primary aim was to explore whether FeNO levels could be used as a screening test for IBD. Our hypothesis was that FeNO levels would be significantly higher in IBD subjects than control subjects without IBD. A second aim was to explore whether FeNO levels directly correlate with individual symptoms and laboratory measures of active IBD. Our hypothesis was that FeNO levels would be higher in subjects experiencing symptoms or labs consistent with active disease.

\section{Materials and Methods}

\section{Sample Population}

The National Health and Nutrition Examination Survey (NHANES) is a major program of the National Center for Health Statistics (NCHS), part of the Centers for Disease Control and
Prevention (CDC), and it has the responsibility for producing vital health statistics for the US population. The yearly survey is unique in that it performs medical interviews, physical examinations and laboratory testing on a nationally representative sample of US citizens. Recently, as part of the NHANES laboratory testing, FeNO levels have been obtained from participants.

\section{Aim 1: Use of FeNO to Screen for IBD}

We restricted our analysis of cases and controls to subjects between the ages of 16-65. For the 2009-2010 iteration of the NHANES, patients were asked whether they have been told they have CD or UC. Those answering yes were included as the study group. Remaining subjects who responded that they were never told they had CD or UC and completed all parts of the questionnaire, laboratory and physiologic measurements were eligible to be controls. We utilized the SPSS 22 (IBM Corporation, Armonk, New York) extension utility bundle "Fuzzy" to perform random draws without replacement to match cases and controls exactly for age and gender.

Several variables were utilized from the NHANES dataset to control for potential confounders in the measurement of FeNO. These included subject height and weight, history of asthma or COPD, participation in vigorous exercise as a proxy for physical fitness, and lifetime smoking history. Wealso controlled for the use of inhaled corticosteroids $\leq 2$ days before testing or consumption of NO-rich foods or beverages $\leq 3$ hours of measuring FeNO.

\section{Aim 2: Use of FeNO in IBD Subjects to Assess Disease Activity}

NHANES does not record IBD disease activity per se and therefore we relied on several available indirect parameters. Clinical symptoms used as a proxy for IBD disease activity included the presence or absence of diarrhea (defined as an average of $\geq 2$ loose bowel movements per day), fatigue, and unintentional weight loss. Laboratory parameters examined to estimate disease activity included anemia $(\leq 10 \mathrm{~g} / \mathrm{dl})$, iron deficiency (ferritin $\leq 20 \mathrm{ng} / \mathrm{ml}$ ), hypoalbuminemia $(\leq 3.2 \mathrm{~g} / \mathrm{dl}$ ), and elevated CRP ( $\geq 1.1 \mathrm{mg} / \mathrm{dl})$.

\section{Fractional Exhalation of Nitric Oxide (FeNO)}

The procedure for FeNO measurement is fully available at (http://wwwn.cdc.gov/nchs/nhanes/2009-2010/ENX_F). In brief, FeNO levels were measured using the Aerocrine NIOX MINO ${ }^{\circ}$, a portable, hand-held NO analyzer (Aerocrine $A B$, Morrisville, NC) approved by the FDA in 2008. This device relies on an electrochemical sensor to detect exhaled NO levels and provides measurements from 5 to $300 \mathrm{ppb}$ in whole numbers. Participants were asked to first empty their lungs, then to place their mouth on the disposable analyzer's filter mouthpiece and to fill their lungs to capacity with NO-free air. Assisted by technicians, participants are then asked to blow out all of their air at a constant pressure. The standard exhalation time, as specified by the manufacturer, was 10 seconds for examinees who were at or above $130 \mathrm{~cm}$ in height, and 6 seconds for those below $130 \mathrm{~cm}$. The NHANES protocol required two valid FeNO measurements 
that were reproducible, in accordance with testing procedures recommended by the manufacturer and similar to those published by the American Thoracic Society and European Respiratory Society. If either or both of the first two valid FeNO measurements was below $30 \mathrm{ppb}$ and the measurements were within $2 \mathrm{ppb}$ of each other, or if both measurements were over $30 \mathrm{ppb}$ and within $10 \%$ of each other, then the test was considered reproducible and complete. If the reproducibility criteria were not met within the first two exhalations, a participant had 2 additional exhalations to satisfy the criteria (up to a total of 4 trials). For analysis, we used the mean of two reproducible measurements.

\section{Procedure for NHANES Complex Sampling}

NHANES utilizes a multi-stage probability sampling design and assigns individual weights to study participants. Weighting takes into account several features of the survey: the specific probabilities of selection for the individual domains that were oversampled, as well as nonresponse and differences between the sample and the total (i.e. US) population. Interviews took place in the home or at a Mobile Exam Center where subjects also undergo laboratory and physiological testing. For this study, we only included subjects who were both interviewed and examined. This represented approximately $98.0 \pm 0.2 \%$ of all NHANES participants for 2009-2010. The Complex Sampling module of SPSS v.22 was utilized to provide accurate error estimates of the weighted data. We designated SDMVSTRA as the "strata" variable, and SDMVPSU as the "clusters" variable. The variable WTMEC2YR was chosen for sample weighting. Sample weight was matched for IBD cases and controls to give an approximate 1:1 ratio. For estimation of parameter error margins, we chose the method of sampling with replacement.

\section{Statistical Analysis}

We first performed an exploratory data analysis to identify miscoded data. We also performed histograms and calculated skewness on weighted continuous data to confirm parametric data distribution. Descriptive and inferential analyses were performed after weighting. For all bivariate calculations of continuous data, we used Student's t-test and crosstabs was performed for categorical data. We first explored whether FeNO could be used to screen a population for IBD. We performed a bivariate analysis comparing the groups and then calculated the area under the receiver operator characteristic (ROC) curve for FeNO. CRP was used as a comparator predictor variable. We performed a multivariate logistic regression analysis to determine if IBD status was an independent predictor of having a FeNO $>25$ ppb after controlling for potential confounders. We then compared FeNO measurements for all subjects with IBD and stratified results by abnormal symptom or lab result. All calculations were performed 2-tailed and measurements of error were calculated (standard deviation, 95\% confidence interval) where appropriate.

\section{Results}

Our 2009-2010 NHANES study group, restricted to ages 16-
65 , consisted of 5,346 participants. Weighted, this represents $199,414,901$ US citizens. The weighted prevalence of IBD was 2,084,895 (1.0\%), including 1,220,442 (58.5\%) females and a mean age $46.7 \pm 13.3$ years. The racial/ethnicity distribution of IBD subjects was: non-Hispanic white $-77.4 \%$, non-Hispanic black - 9.1\%, and Hispanic/Mexican - 12.3\%.

\section{Aim 1: Use of FeNO to Screen for IBD}

Table 1 shows the characteristics of the subjects stratified by IBD status. Our demographic matching process was successful. As expected, subjects with IBD had a higher prevalence of diarrhea, unintentional underweight status, and fatigue. On labs, IBD subjects had lower levels of ferritin and higher levels of CRP. There were no differences in albumin or hemoglobin levels. With regards to potential confounders when measuring FeNO, those subjects with IBD had a higher use of inhaled corticosteroids before the test, corresponding to a higher prevalence of chronic pulmonary disorders. Although a higher percentage of controls ever smoked, there were more current smokers in the IBD group.

Mean levels of FeNO for IBD cases and controls were nearly identical, $(17.0 \pm 16.2$ vs. $16.7 \pm 14.5 \mathrm{ppb})$. The proportion of subjects with the highest quartile of FeNO ( $\geq 19.1 \mathrm{ppb})$ was actually higher in the controls vs. those with IBD (33.7 vs. $23.9 \%$ ). A value $\geq 25 \mathrm{ppb}$ is established as a cutoff in the asthma literature [14]. Nearly twice as many subjects without IBD had a value above that threshold (18.6 vs. 9.7\%) compared to those with IBD. By multivariate logistic regression analysis the odds of having a FeNO > 25 ppb was half (OR=0.501; 95\% CI 0.4970.504 ) for subjects with IBD compared to those without IBD after controlling for age, gender, smoking and asthma/COPD status, height, weight, and NO-rich meal before testing. Another way to evaluate the discriminant value of FeNO for identifying subjects with IBD is to determine the area under ROC for that parameter. Figure 1 demonstrates that FeNO performed no better than chance (0.5) and was inferior to CRP.

\section{Aim 2: Determining Whether FeNO Can Identify Active Disease}

Figure 2 includes only subjects with IBD. Those individuals with diarrhea, fecal urgency, and fatigue had higher FeNO levels. Individuals self-defined as unintentionally underweight, however, had far lower FeNO levels. For lab parameters, in theory associated with more active disease, FeNO levels were uniformly higher on average for those with values in the normal range.

\section{Discussion}

Using a population-based sample, we were unable to reject the null hypothesis for the use of FeNO as a test to discriminate individuals with and without IBD. Additionally, in a group of subjects with known IBD, FeNO levels were not higher in patients with laboratory values suggestive of active disease. FeNO levels were higher in IBD patients with diarrhea, rectal urgency, and fatigue but were lower in those with unintentional weight loss. As such, FeNO appears to not be a useful test for the evaluation of IBD. 
Table 1: Characteristics of IBD Patients and Controls.

\begin{tabular}{|c|c|c|c|}
\hline \multicolumn{4}{|c|}{ Weighted $\mathbf{n}$} \\
\hline & $\begin{array}{c}\text { Total } \\
\mathrm{n}=4,098,796\end{array}$ & $\begin{array}{l}\text { IBD Subjects } \\
\mathrm{n}=\mathbf{2 , 0 8 4 , 8 9 5}\end{array}$ & $\begin{array}{c}\text { Controls } \\
n=2,013,901\end{array}$ \\
\hline Age, y $( \pm$ SD) & 46.7 (13.3) & 46.7 (13.3) & $46.7(13.5)$ \\
\hline Gender ( $\%$ female) & 59.7 & 58.5 & 60.9 \\
\hline $\begin{array}{c}\text { Race (\%) } \\
\text { non-Hispanic white } \\
\text { non-Hispanic black } \\
\text { Hispanic }\end{array}$ & $\begin{array}{c}71.9 \\
9.9 \\
12.8\end{array}$ & $\begin{array}{c}77.4 \\
9.1 \\
12.3\end{array}$ & $\begin{array}{l}66.2 \\
10.6 \\
13.3\end{array}$ \\
\hline Ratio Income: Poverty Level $( \pm$ SD) & $3.2(1.7)$ & $3.0(1.6)$ & $3.4(1.8)$ \\
\hline Education beyond high school (\%) & 41.0 & 40.5 & 41.5 \\
\hline \multicolumn{4}{|c|}{ IBD Activity Parameters } \\
\hline Diarrhea $(\%)^{*}$ & 5.7 & 9.0 & 2.3 \\
\hline Underweight without intention (\%) & 2.4 & 4.7 & 0.0 \\
\hline Generalized Fatigue (\%) & 59.7 & 71.9 & 47.6 \\
\hline Hemoglobin, g/dl ( \pm SD) & $14.4(1.3)$ & $14.5(1.4)$ & $14.4(1.3)$ \\
\hline Ferritin (ng/ml) & $86.29(64.3)$ & $70.2(27.9)$ & $101.5(82.7)$ \\
\hline C-reactive protein, $\mathrm{mg} / \mathrm{dl}( \pm \mathrm{SD})$ & $0.35(0.44)$ & $0.42(0.48)$ & $0.28(0.38)$ \\
\hline Albumin, g/dl ( \pm SD) & $4.2(0.3)$ & $4.2(0.3)$ & $4.2(0.4)$ \\
\hline \multicolumn{4}{|c|}{ FeNO Measurement Parameters } \\
\hline Body weight, lbs. ( \pm SD) & $176.3(45.0)$ & $177.0(42.7)$ & $175.6(47.4)$ \\
\hline Body Height, inch $( \pm$ SD) & $66.3(3.7)$ & $66.3(3.6)$ & $66.3(3.7)$ \\
\hline Ate NO-rich food 3 hours before measurement (\%) & 8.4 & 8.3 & 8.5 \\
\hline Used inhaled steroid $\leq \mathbf{2} \mathrm{d}$ before measurement (\%) & 9.5 & 14.5 & 4.7 \\
\hline Asthma/COPD/Chronic Bronchitis (\%) & 26.8 & 30.9 & 22.6 \\
\hline Participate in vigorous exercise (\%) & 20.0 & 18.7 & 21.2 \\
\hline Lifetime use $>100$ cigarettes $(\%)$ & 49.6 & 46.6 & 52.6 \\
\hline Current smoking (\%) & 48.0 & 54.8 & 41.6 \\
\hline
\end{tabular}

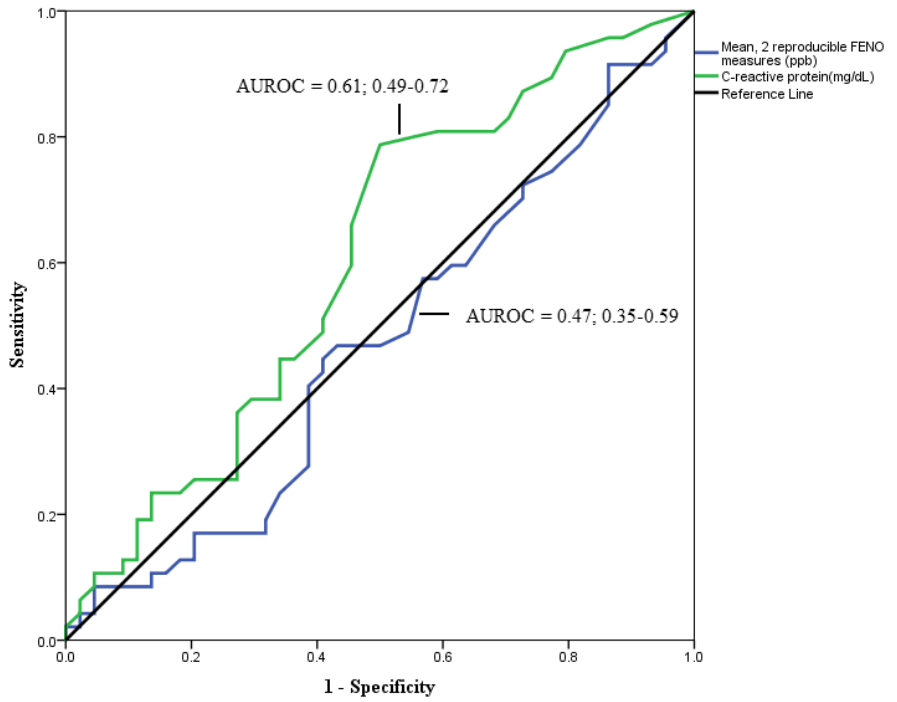

Figure 1: Area under the receiver operator characteristic curve (with $95 \% \mathrm{CI}$ ) for $\mathrm{FeNO}$ as a predictor of IBD status. CRP included as a reference. 


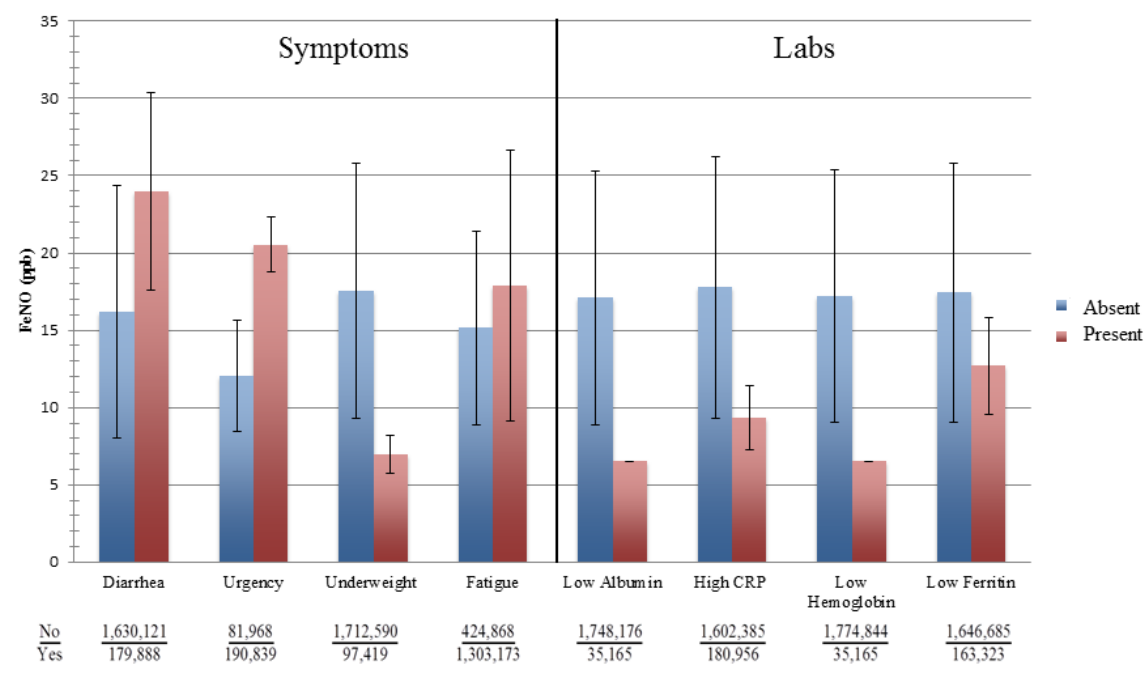

Figure 2: Relationship between symptoms and signs of IBD and FeNO levels in subset of subjects with IBD.

FeNO levels can be affected by several factors including exhalation flow rate, nasal NO contamination, the NO analyzer used, age, height, current smoking, and anti-inflammatory medications. Less studied factors include gender, weight and pretest diet [14]. The role of FeNO has been established in diagnosing asthma and can aid in predicting which patients may respond better to corticosteroid treatment [14]. There is evidence that eosinophilic airway inflammation increases FeNO levels by inducing NOS2 (nitric oxide synthase 2) expression or activity via specific interleukins [10]. In the largest study $(n=566)$ to date, a positive correlation was found between FeNO and sputum eosinophil count $\left(\mathrm{r}^{2}=0.26, \mathrm{P}<0.001\right)[9,15]$. Furthermore, FeNO of $36 \mathrm{ppb}$ had a sensitivity of $78 \%$ and specificity of $72 \%$ for sputum eosinophilia of $>3 \%$. In another study by Shaw and colleagues, a FeNO of $<26 \mathrm{ppb}$ had a negative predictive value of $85 \%$ for sputum eosinophils $<3 \%$ [16]. Interestingly, few studies point to the role of mucosal eosinophilia, yet this usually represents only a small percentage of the infiltrating leukocytes [17].

Previous studies by Quenon, etal. and Koek, etal.demonstrated higher FeNO levels in patients with active IBD compared to those with inactive disease and healthy controls. In contrast, our study found a trend toward lower values especially in those with laboratory parameters consistent with active disease $[11,12]$. For example, mean FeNO levels were lower in IBD subjects with a CRP $\geq 1.1 \mathrm{mg} / \mathrm{dl}$ compared to those with a value below this cutpoint ( $9.3 \pm 4.2$ vs. $17.7 \pm 16.9 \mathrm{ppb})$. Additionally, IBD subjects had nearly the same FeNO level as those without IBD (17.0 \pm 16.2 vs. $16.7 \pm 14.5 \mathrm{ppb}$ ). Our IBD subjects were well-matched with controls on several parameters known to affect FeNO levels. Asthma, a factor which should increase FeNO levels, was more common in IBD subjects and should have biased the results in the expected direction. This effect may have been attenuated by higher inhaled corticosteroid use in IBD subjects. These variances were controlled for in the statistical analysis. The most likely explanation for our finding is that GI luminal mucosal activity is simply not reflected in the FeNO.

When looking at FeNO levels in subjects with known IBD, the presence of abnormal laboratory tests did not correlate with higher FeNO level. This is in contrast to what would be expected if FeNO were a good marker of active IBD. While one explanation for this finding would be that these lab values were not a good indication of disease activity, they are all common, accepted findings of patients with active IBD. A more likely explanation is that FeNO does not adequately predict disease activity.

One aim was to examine if FeNO could be used as a noninvasive screening test for IBD. The rationale for this investigation is that current modalities are inadequate. Traditional blood tests ordered when considering a diagnosis of IBD usually include the ESR and CRP. A pediatric study by Beattie, et al. found that at the time of diagnosis, $100 \%$ of CD patients but only $60 \%$ of UC patients had increased CRP compared with none of the children without IBD. ESR proved to be the second best marker, with $85 \%$ of $\mathrm{CD}$ and $23 \%$ of UC patients positive compared to none of the children in the control group [18]. More commonly, CRP and ESR are used to non-invasively monitor disease activity. A prospective study by Fagan, et al. measured serum levels of both markers in 64 patients with CD and 50 with UC and compared the results with the clinical assessment of disease activity. They found that CRP values did correlate with disease activity. ESR was also higher in CD but did not closely reflect disease activity in individual patients [19]. The GETAID group further looked at both of these markers in predicting disease relapse and found that when combined they had a sensitivity of $89 \%$ but a specificity of only 43\% [20]. Serologic markers including perinuclear antineutrophil cytoplasmic antibody (pANCA) and anti-Saccharomyces cerevisiae antibody (ASCA) have been used for many years to screen and classify patients with possible 
IBD. Peeters, et al. found that when these markers are used for differentiating patients with IBD from healthy controls they had $50-60 \%$ sensitivity and $91-95 \%$ specificity [21]. A third antibody, anti-OmpC, has been shown to have low sensitivity for both CD (24\%) and UC (11\%); however, when the three antibodies are combined in a panel, the overall sensitivity was $65-75 \%$ and specificity $94 \%$ for diagnosing IBD when one or more is found to be present [22]. Genetic testing has identified only the nucleotide binding oligomerization domain protein 2 (NOD2) as a meaningful predictor for CD [23]. A population-based, casecontrol study of NOD2 mutations found that $14 \%$ of healthy controls carried a mutation compared to $37 \%$ of $\mathrm{CD}$ patients [24]. Currently, Prometheus Labs, Inc. (San Diego, CA) offers the IBD sgi Diagnostic ${ }^{\mathrm{TM}}$ which assesses 18 serologic, genetic and inflammatory markers in one blood test to differentiate IBD vs. non-IBD and to clarify IBD-subtype (CD vs. UC). Using their Smart Diagnostic Algorithm, the company reports sensitivity values for IBD of $74 \%$, CD $89 \%$ and UC $98 \%$ and specificity values for IBD 90\%, CD 81\% and UC 83\% (Prometheus Lab data).

Stool testing, including fecal calprotectin and lactoferrin, is a more recent tool both to differentiate IBD from non-IBD patients and to non-invasively assess disease activity. Calprotectin is stable in stool samples for up to seven days at room temperature making it a reliable measurement of active intestinal inflammation [25]. In one meta-analysis, the presence of fecal calprotectin was 93\% sensitive and $96 \%$ specific for identifying patients with IBD [26]. Lactoferrin is used similarly to fecal calprotectin. In a study of 104 CD patients, 80 UC patients, 31 IBS patients and 56 healthy controls the mean fecal lactoferrin value ( $\mu \mathrm{g} / \mathrm{g}$ fecal weight) was $440 \pm 128$ for CD patients, $1125 \pm 498$ for UC patients, $1.27 \pm 0.29$ for IBS patients, and $1.45 \pm 0.4$ for healthy controls [27]. Fecal lactoferrin was $90 \%$ specific in identifying inflammation in IBD and $100 \%$ specific in ruling out IBS.

Weaknesses of our study may explain why our findings are inconsistent with previous studies. For example, we did not have access to traditional measures of disease activity such as the CDAI and relied on non-specific symptoms and labs. Similarly, NHANES does not provide endoscopic data and therefore mucosal disease activity was unknown. Moreover, without biopsy data, the extent of mucosal eosinophilia is not known. An additional confounder is the use of anti-TNF, corticosteroid, and immunomodulator therapy which potentially could reduce FeNO levels by down-regulating inducible nitric oxide synthase. Finally, misclassification is an important consideration; subjects were asked if they had IBD but this was not confirmed with clinical data. Subject reported data is a problem universal to database research like ours; however, we would expect that participants in a large, nationwide survey like NHANES would be able to appropriately categorize themselves into having or not having IBD.

In summary, we did not find that FeNO levels could be used clinically to identify individuals with IBD vs. those without the disease. Similarly, in individuals with IBD, we did not find that FeNO could be used as a marker of disease activity. Though we acknowledge potential weaknesses of using our dataset, it does represent an unbiased sample of US citizens, and we did control for important known confounders. Measurement of FeNO requires a significant amount of resources as well as patient cooperation relative to available non-invasive alternatives. Until there is more confirmatory evidence of its utility, we recommend that it not be used to screen for IBD or assess disease activity.

\section{Acknowledgements}

We acknowledge funding assistance from award K24DK083268 from the DHHS to FKF.

\section{References}

1. Abraham C, Cho JH. Inflammatory bowel disease. N Engl J Med. 2009;361(21):2066-78. doi: 10.1056/NEJMra0804647.

2. Vermeire S, Van Assche G, Rutgeerts P. Laboratory markers in IBD: useful, magic, or unnecessary toys? Gut. 2006;55(3):426-431.

3. D'Haens G, Ferrante M, Vermeire S, Baert F, Noman M, Moortgat L, et al. Fecal calprotectin is a surrogate marker for endoscopic lesions in inflammatory bowel disease. Inflamm Bowel Dis. 2012;18(12):22182224. doi: $10.1002 /$ ibd.22917.

4. Cross RK, Wilson KT. Nitric oxide in inflammatory bowel disease. Inflamm Bowel Dis. 2003 May;9(3):179-189.

5. Ljung T, Beijer E, Herulf M, Weitzberg E, Lundberg JO, Finkel Y, et al. Increased rectal nitric oxide in children with active inflammatory bowel disease. J Pediatr Gastroenterol Nutr. 2002;34(3):302-306.

6. Lundberg JO, Hellstrom PM, Lundberg JM, Alving K. Greatly increased luminal nitric oxide in ulcerative colitis. Lancet. 1994;344(8938):16731674.

7. American Thoracic Society, European Respiratory Society. ATS/ERS recommendations for standardized procedures for the online and offline measurement of exhaled lower respiratory nitric oxide and nasal nitric oxide, 2005. Am J Respir Crit Care Med. 2005;171(8):912930.

8. Lim KG, Mottram C. The use of fraction of exhaled nitric oxide in pulmonary practice. Chest. 2008;133(5):1232-1242. doi: 10.1378/ chest.07-1712.

9. Berry M, Morgan A, Shaw DE, Parker D, Green R, Brightling C, et al. Pathological features and inhaled corticosteroid response of eosinophilic and non-eosinophilic asthma. Thorax. 2007;62(12):10431049.

10. Paoliello-Paschoalato AB, Oliveira SH, Cunha FQ. Interleukin 4 induces the expression of inducible nitric oxide synthase in eosinophils. Cytokine. 2005;30(3):116-124.

11. Quenon L, Hindryckx P, De Vos M, De Looze D, Joos G, Brusselle G, et al. Hand-held fractional exhaled nitric oxide measurements as a noninvasive indicator of systemic inflammation in Crohn's disease.J Crohns Colitis. 2013;7(8):644-648. doi: 10.1016/j.crohns.2012.09.021.

12. Koek GH, Verleden GM, Evenepoel P, Rutgeerts P. Activity related increase of exhaled nitric oxide in Crohn's disease and ulcerative colitis: a manifestation of systemic involvement? Respir Med. 2002;96(7):530-535

13. Ozyilmaz E, Yildirim B, Erbas G, Akten S, Oguzulgen IK, Tunc B, et al. Value of fractional exhaled nitric oxide (FE NO) for the diagnosis of 
pulmonary involvement due to inflammatory bowel disease. Inflamm Bowel Dis. 2010;16(4):670-676. doi: 10.1002/ibd.21085.

14. Dweik RA, Boggs PB, Erzurum SC, Irvin CG, Leigh MW, Lundberg JO, et al. An official ATS clinical practice guideline: interpretation of exhaled nitric oxide levels (FENO) for clinical applications. Am J Respir Crit Care Med. 2011;184(5):602-615. doi: 10.1164/rccm.9120-11ST.

15. Berry MA, Shaw DE, Green RH, Brightling CE, Wardlaw AJ, Pavord ID. The use of exhaled nitric oxide concentration to identify eosinophilic airway inflammation: an observational study in adults with asthma. Clin Exp Allergy. 2005;35(9):1175-1179.

16. Shaw DE, Berry MA, Thomas M, Green RH, Brightling CE, Wardlaw AJ et al. The use of exhaled nitric oxide to guide asthma management: a randomized controlled trial. Am J Respir Crit Care Med. 2007;176(3):231-237.

17. Rothenberg ME. Eosinophilic gastrointestinal disorders (EGID). Allergy Clin Immunol. 2004 Jan;113(1):11-28.

18. Beattie RM, Walker-Smith JA, Murch SH. Indications for investigation of chronic gastrointestinal symptoms. Arch Dis Child. 1995;73(4):354355

19. Fagan EA, Dyck RF, Maton PN, Hodgson HJ, Chadwick VS, Petrie A, et al. Serum levels of C-reactive protein in Crohn's disease and ulcerative colitis. Eur J Clin Invest. 1982;12(4):351-359.

20. Consigny Y, Modigliani R, Colombel JF, Dupas JL, Lemann M, Mary JY, et al. A simple biological score for predicting low risk of short-term relapse in Crohn's disease. Inflamm Bowel Dis. 2006;12(7):551-557.

21. Peeters M, Joossens S, Vermeire S, Vlietinck R, Bossuyt X, Rutgeerts P.
Diagnostic value of anti-Saccharomyces cerevisiae and antineutrophil cytoplasmic autoantibodies in inflammatory bowel disease. Am J Gastroenterol. 2001 ;96(3):730-734.

22.Zholudev A, Zurakowski D, Young W, Leichtner A, Bousvaros A. Serologic testing with ANCA, ASCA, and anti-OmpC in children and young adults with Crohn's disease and ulcerative colitis: diagnostic value and correlation with disease phenotype. Am J Gastroenterol. 2004;99(11):2235-41.

23. Cho JH, Brant SR. Recent insights into the genetics of inflammatory bowel disease. Gastroenterology. 2011;140(6):1704-1712. doi: 10.1053/j.gastro.2011.02.046.

24. Brant SR, Wang MH, Rawsthorne P, Sargent M, Datta LW, Nouvet F, et al. A population-based case-control study of CARD15 and other risk factors in Crohn's disease and ulcerative colitis. Am J Gastroenterol. 2007;102(2):313-323.

25. Roseth AG, Aadland E, Jahnsen J, Raknerud N. Assessment of disease activity in ulcerative colitis by faecal calprotectin, a novel granulocyte marker protein. Digestion. 1997;58(2):176-80.

26. van Rheenen PF, Van de Vijver E, Fidler V. Faecal calprotectin for screening of patients with suspected inflammatory bowel disease: diagnostic meta-analysis. BMJ. 2010;341:c3369. doi: 10.1136/bmj. c3369.

27. Kane SV, Sandborn WJ, Rufo PA, Zholudev A, Boone J, Lyerly D, et al. Fecal lactoferrin is a sensitive and specific marker in identifying intestinal inflammation. Am J Gastroenterol. 2003 Jun;98(6):13091314. 